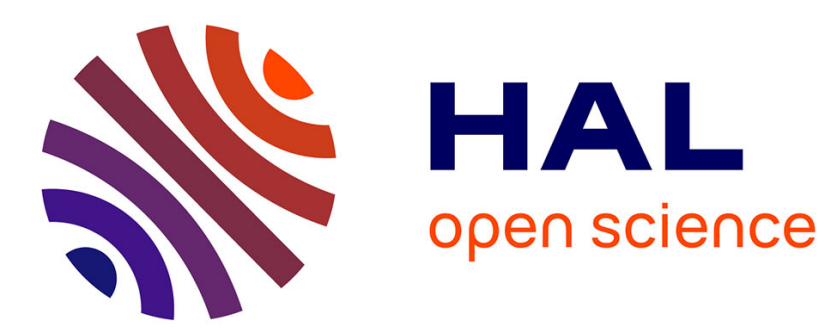

\title{
Avalanche Polynomials of some Families of Graphs
}

Dominique Rossin, Arnaud Dartois, Robert Cori

\section{To cite this version:}

Dominique Rossin, Arnaud Dartois, Robert Cori. Avalanche Polynomials of some Families of Graphs.

Trends in Mathematics, 2004, Mathematics and COmputer Science III, pp.81-94. hal-00016371

\section{HAL Id: hal-00016371 \\ https://hal.science/hal-00016371}

Submitted on 2 Jan 2006

HAL is a multi-disciplinary open access archive for the deposit and dissemination of scientific research documents, whether they are published or not. The documents may come from teaching and research institutions in France or abroad, or from public or private research centers.
L'archive ouverte pluridisciplinaire HAL, est destinée au dépôt et à la diffusion de documents scientifiques de niveau recherche, publiés ou non, émanant des établissements d'enseignement et de recherche français ou étrangers, des laboratoires publics ou privés. 


\title{
Avalanche polynomials of some families of graphs
}

\author{
R. Cori, A. Dartois and D. Rossin
}

\begin{abstract}
We study the abelian sandpile model on different families of graphs. We introduced the avalanche polynomial which enumerates the size of the avalanches triggered by the addition of a particle on a recurrent configuration. This polynomial is calculated for several families of graphs. In the case of the complete graph, the result involves some known result on Parking functions [12, 11].

Bak, Tang and Wiesenfeld [2] introduced 15 years ago the concept of self organized criticality which allowed to describe a large variety of physical systems like earthquakes [5, 14., forest fires and even some fluctuations in the stock market [1]. One version of this concept is the sandpile cellular automaton model which uses a 2 dimensional lattice; in the sites of this lattice particles are added giving rise to a toppling when their number in a site exceeds a given bound. A toppling on a site may be followed by the toppling on one or more of its neighbors and this sequence of topplings is called an avalanche. Many authors have studied the distribution of the sizes (the number of topplings performed) of the avalanches for this model showing that they obey to power-laws [10, 7, 13].

The sandpile model was also considered by combinatorists as a game on a graph called the chip firing game [3, 4]. Relationships between the structure of the graph and the recurrent configurations of the physical model were pointed out [6, 9].

Experiments on the distribution of sizes of the avalanches were considered only for the 2 dimensional lattice and for some classes of regular graphs. Very little is known for arbitrary graphs [8]. In this paper, a polynomial, encoding the avalanche sizes obtained by adding a particle to a site in a recurrent configuration, is associated to a graph. We determine this polynomial for various families of graphs.

These families are the trees, the cycles, the complete graphs and the lollypop graphs. For these families of graphs the power law observed for the 2 dimensional grid is no more satisfied. The computation of the avalanche polynomial of the complete graph uses a bijection between recurrent configurations of this graph and the so-called parking functions. This computation allows the determination of the avalanche distributions on the lollypop graphs which shows out the existence of peaks also observed in some other families of regular graphs.
\end{abstract}

\section{Recurrent configurations of the sandpile model}

In this section we recall the main results on the sandpile model which are useful in this paper.

In what follows $G=(X, E)$ is a connected multigraph with $n+1$ vertices:

$$
X=\left\{x_{1}, x_{2}, \ldots, x_{n}, x_{n+1}\right\},
$$

vertex $x_{n+1}$ is distinguished and called the sink. A configuration of the sandpile model in this graph is a sequence of $n$ integers

$$
u=\left(u_{1}, u_{2}, \ldots, u_{n}\right) .
$$


For a configuration $u$, the integer $u_{i}$ will be considered as a number of particles placed on the vertex $x_{i}$.

A configuration is stable if the integers $u_{i}$ satisfy $0 \leq u_{i}<d_{i}$, where $d_{i}$ is the degree of the vertex $x_{i}$. In a configuration which is not stable a vertex $x_{i}$ with $u_{i} \geq d_{i}$ may perform a toppling giving a new configuration $v$ such that $v_{i}=u_{i}-d_{i}$ and $v_{j}=u_{j}+\varepsilon_{i, j}$, where $\varepsilon_{i, j}$ denotes the number of edges between vertices $x_{i}$ and $x_{j}$ in the multigraph $G$.

We will write the toppling of vertex $x_{i}$ by:

$$
u \rightarrow_{i} v .
$$

An avalanche is a sequence of topplings; we will use the notation

$$
u \stackrel{*}{\rightarrow} v \text {. }
$$

for a configuration $v$ which is reached from $u$ after an avalanche.

The size of the avalanche is the number of topplings performed. In the Figure 11 below is given an avalanche of size 3, the sink is represented by a black vertex.

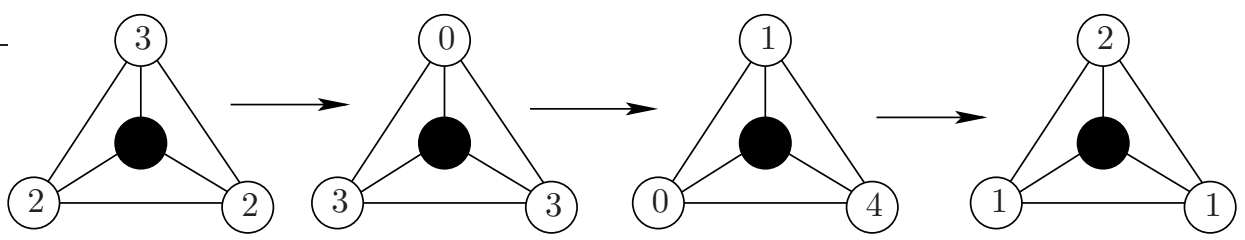

Figure 1: An avalanche on $K_{4}$

In the sandpile model the main operation consists in:

1. taking a stable configuration

2. adding a particle on one of the vertices

3. performing topplings until a new stable configuration is obtained.

The above example is an illustration of this operation for the graph $K_{4}$ and the configuration $(2,2,2)$. It is not difficult to prove that the stable configuration obtained after a sequence of topplings from a given unstable configuration does not depend on the order in which these topplings are performed.

The recurrent configurations of the sandpile model are the stable configurations which are met infinitely often when performing the above operations 2 and 3 . Note that not all the stable configurations are recurrent. The recurrent configurations play a key role in the sandpile model; their number is the tree number of the underlying graph, hence independent of the vertex chosen as the sink.

There are many characterizations of recurrent configurations and many structural results on them; in the sequel we will simply use the following characterization. Let $\pi$ denotes the configuration such that $\pi_{j}$ is the number of edges between vertex $x_{j}$ and the sink.

Proposition 1. The configuration $u$ is recurrent if and only if it is stable and

$$
\pi+u \stackrel{*}{\rightarrow} u,
$$


where $\pi+u$ denotes the configuration $v$ such that $v_{i}=u_{i}+\pi_{i}$ for $1 \leq i \leq n$.

Moreover in this avalanche every vertex of $G$ topples exactly once.

\section{Avalanche polynomials: some simple examples}

Let $u$ be a recurrent configuration on the multigraph $G=(X, E)$, we consider the avalanche obtained when adding a particle on site $x_{i}$, we will call it a principal avalanche, we denote by $a d_{G}\left(u, x_{i}\right)$ the size of this principal avalanche. Note that a principal avalanche may be of size 0 . This is the case when the particle is added on a site $i$ such that $u_{i}<d_{i}-1$.

The three recurrent configurations of the cycle $C_{3}$ are given below:
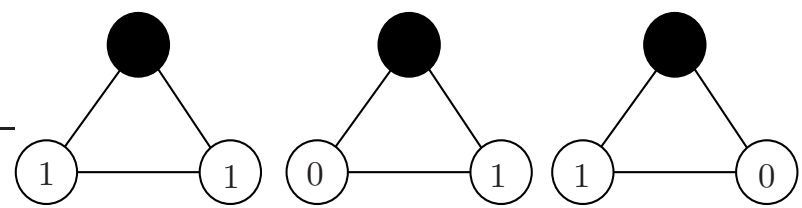

Figure 2: Recurrent configurations on $C_{3}$.

The sizes of the principal avalanches are 2,2 for the first recurrent configuration, 1,0 and 0,1 for the two other configurations.

To any connected graph $G=(X, E)$ with a sink, we associate a polynomial enumerating the sizes of the principal avalanches. This polynomial is given by:

$$
A v_{G}(x)=\sum \alpha_{k} x^{k},
$$

where $\alpha_{k}$ is the number of principal avalanches of size $k$.

For instance from the example on Figure 2 we obtain:

$$
A v_{C_{3}}(x)=2+2 x+2 x^{2} .
$$

Note that for any graph $G, A v_{G}(1)$ is equal to $n$ times the tree number of $G$. Thus it is independent of the sink chosen for $G$. Note that indeed, the polynomial $A v_{G}$ is dependent of this sink.

\subsection{Avalanche polynomials of trees}

If $G$ is a tree $T$ then it has only one recurrent configuration, this configuration $u_{T}$ is such that $u_{T i}=d_{i}-1$ for each vertex $x_{i}$. It is convenient to draw the tree in such a way that the sink is the root of the tree.

Adding a particle on vertex $x_{i}$ gives a sequence of topplings on all the vertices of the subtree of $T$ rooted at $x_{i}$, the avalanche ends there if $x_{i}$ is a son of the root. If $x_{i}$ is not a son of the root, then after this first sequence of topplings the father $x_{j}$ of $x_{i}$ in $T$ gets one particle and a new sequence of topplings can be performed. Hence we have:

$$
a d_{T}\left(u_{T}, x_{i}\right)=a d_{T}\left(u_{T}, x_{j}\right)+t_{i},
$$

where $t_{i}$ is the number of vertices of the subtree with root $x_{i}$. 
An example of the computation of avalanche sizes on a tree is given below; on each vertex is indicated the size of the avalanche obtained when adding a particle on it.

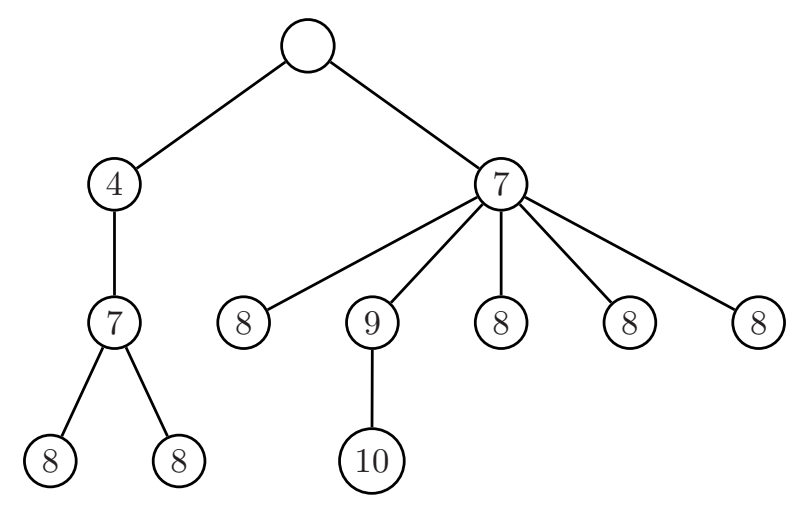

Figure 3: A tree $T_{1}$ and the sizes of the principal avalanches.

The avalanche polynomial on this tree is:

$$
A v_{T_{1}}(x)=x^{4}+2 x^{7}+6 x^{8}+x^{9}+x^{10} .
$$

Note that $A v_{G}(0)=0$ if and only if $G$ is a tree. Indeed a graph which is not a tree has more than one recurrent configuration and necessarily at least one of them has a vertex $x_{i}$ with $u_{i}<d_{i}-1$; for this vertex, adding a particle does not produce any toppling.

The avalanche polynomial does not characterize the tree since the avalanche polynomial of the tree $T_{2}$ below is also $A v_{T_{1}}(x)^{1}$.

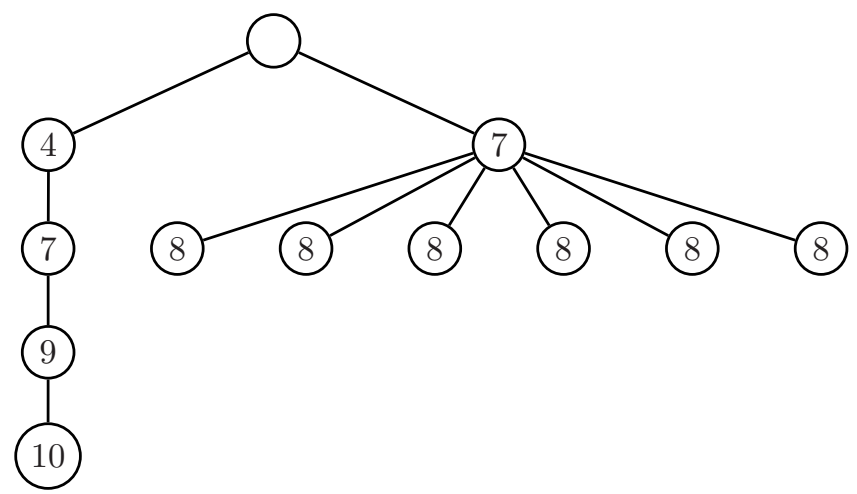

Figure 4: A tree $T_{2}$ with the same avalanche polynomial as $T_{1}$.

\footnotetext{
${ }^{1}$ this example is due to Michel Marcus.
} 
When we choose a different root, the avalanche polynomial changes also. From any tree or graph, we can define the set of avalanche polynomials for all different possible roots. This set is neither characteristic of the tree nor the graph. Indeed, the trees $T$ and $T^{\prime}$ of Figure 6 built from $T_{1}$ and $T_{2}$ are not isomorphic but admit the same set of avalanche polynomials, when the root spans the set of vertices.

The set $A v^{\mathcal{T}}$ of polynomials $P$ such that there exists a tree $T$ satisfying $A v_{T}=P$ is the smallest set such that:

$$
\begin{aligned}
& x \in A v^{\mathcal{T}} \\
& P, Q \in A v^{\mathcal{T}} \quad \Longrightarrow \quad P+Q \in A v^{\mathcal{T}} \\
& P \in A v^{\mathcal{T}} \quad \Longrightarrow \quad x^{a}(P+1) \in A v^{\mathcal{T}} \text {, where } a=P(1)+1 \text {. }
\end{aligned}
$$

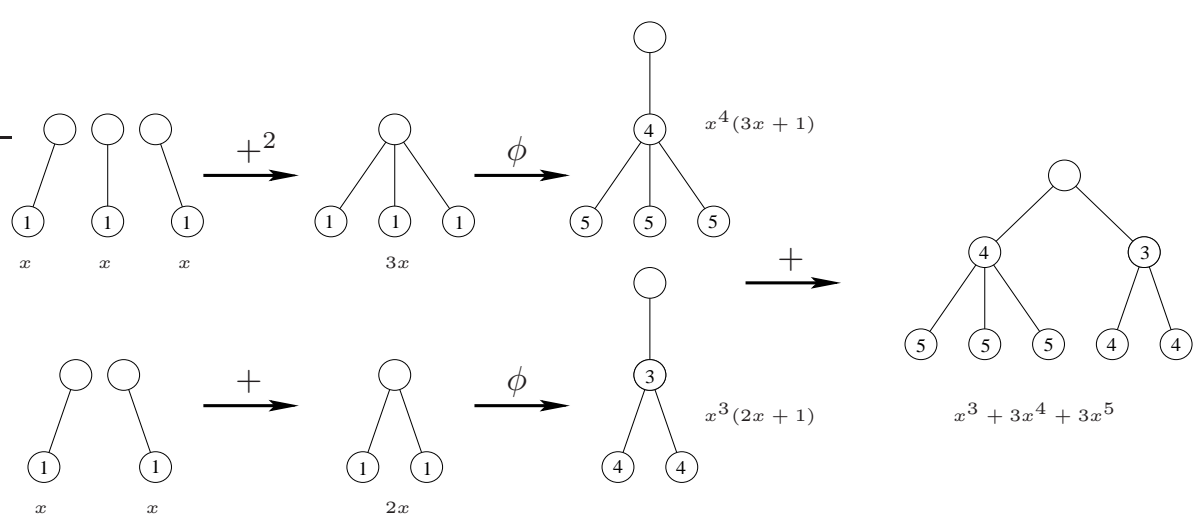

Figure 5: Inductive computation of the avalanche polynomial of a tree.

The proof is quite straightforward, since the two operations (2.2) and (2.3) correspond to two basic operations on trees (cf Figure 5): sticking two trees by merging their root $(+)$, and adding a new root only connected to the old one $(\phi)$. The result follows by the fact that any rooted tree can be obtained by these two operations starting from the two-vertices tree (whose avalanche polynomial is $x$ ).

By the means of the two operations + and $\Phi$, we can show that $T$ and $T^{\prime}$ have the same set of avalanche polynomials. We note $x_{0}$ (resp. $y_{0}$ ) the root of the tree $T$ (resp. $T^{\prime}$ ), and $x_{1}$ (resp. $x_{2}$ ) the root of its left sub-tree (resp. right sub-tree). The vertices defined in the same way on $T^{\prime}$ are called $y_{1}$ and $y_{2}$. Among the other vertices of $T$, we note $x_{3}, x_{4}, \ldots, x_{13}$ (resp. $x_{25}, x_{26}, \ldots, x_{35}$ ) the ones belonging to the first copy of $T_{1}$ (resp. $T_{2}$ ) and $x_{14}, x_{15}, \ldots, x_{24}\left(\right.$ resp. $\left.x_{36}, x_{37}, \ldots, x_{46}\right)$ the ones belonging to the second copy of $T_{1}$ (resp. $T_{2}$ ). The corresponding vertices of $T^{\prime}$ are called $y_{i}$, where the index $i$ is defined by the same manner (cf. Figure 6). Then, we can show that the avalanche polynomial of $T$ rooted in $x_{i}$ equals the avalanche polynomial of $T^{\prime}$ rooted in $y_{i}$ for all indices $i$.

If $x_{0}$ is chosen as the root of $T$, we choose $y_{0}$ as root of $T^{\prime}$. Then $T=$ $\Phi\left(T_{1}+T_{1}\right)+\Phi\left(T_{2}+T_{2}\right)$ and $T^{\prime}=\Phi\left(T_{1}+T_{2}\right)+\Phi\left(T_{1}+T_{2}\right)$. In term of polynomials, we get $A v_{T}=A v_{T^{\prime}}$, since $A v_{T_{1}}=A v_{T_{2}}$. 


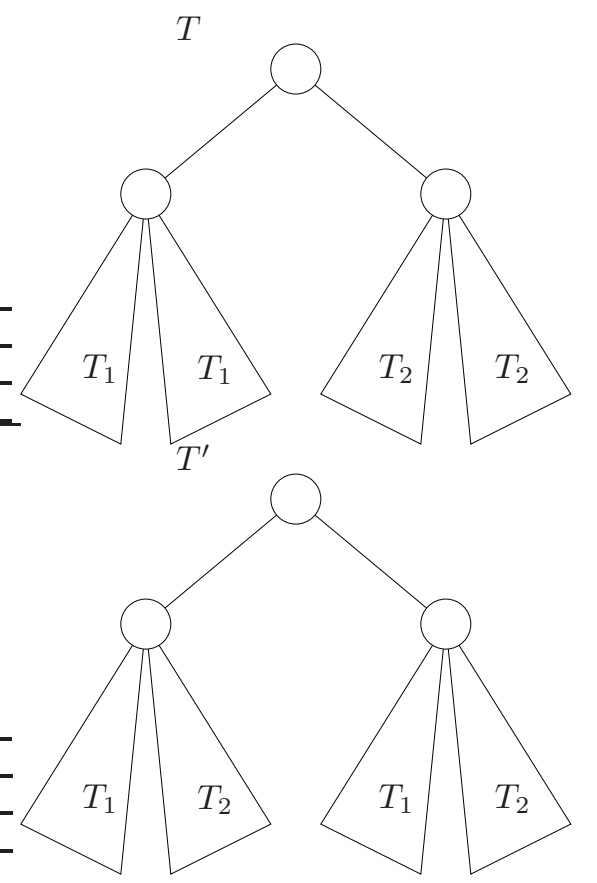

Figure 6: Trees $T$ and $T^{\prime}$.

By symmetry of $\left(T, T^{\prime}\right)$ in regards of $T_{1}$ and $T_{2}$, it is sufficient to show that $A v_{T}=A v_{T^{\prime}}$ when $x_{i}$ (resp. $y_{i}$ ) is chosen as root of $T$ (resp. $T^{\prime}$ ), only for indices $i=1$ or $3 \leq i \leq 24$.

If $x_{1}$ is chosen as root of $T$, we choose $y_{1}$ as root of $T^{\prime}$. This time, $T=$ $T_{1}+T_{1}+\Phi^{2}\left(T_{2}+T_{2}\right)$ and $T^{\prime}=T_{1}+T_{2}+\Phi^{2}\left(T_{1}+T_{2}\right)$. But, in term of polynomials, we still get $A v_{T}=A v_{T^{\prime}}$.

Let choose $x_{i}$ (resp. $y_{i}$ ) as root of $T$ (resp. $T^{\prime}$ ), with $3 \leq i \leq 13$. Then, it exists a sequence $s$ of operations + and $\Phi$ that build $T_{1}$ rooted in $x_{i}$ starting from $\left\{x_{1}\right\}$. If we set $A v_{A}=0$ for the tree $A$ with one vertex, then, in term of polynomials, the sequence $s$ applied to 0 leads to the avalanche polynomials of $T_{1}$ rooted in $x_{i}$. Hence we have $T=s\left(T 1+\Phi^{2}\left(T_{2}+T_{2}\right)\right)$ and also $T^{\prime}=s\left(T 2+\Phi^{2}\left(T_{1}+T_{2}\right)\right)$. Consequently, $A v_{T}=A v_{T^{\prime}}$. If $14 \leq i \leq 24$, the same argument is possible, and we also get the wanted result.

Hence, $T$ and $T^{\prime}$ are non-isomorphic but have the same set of avalanche polynomials.

\subsection{Avalanche polynomials of cycles}

Another simple example on which the avalanche polynomial can be computed easily is that of the cycle $C_{n+1}$; we have the following result:

Proposition 2. There exists a principal avalanche of size $k$ on $C_{n+1}$ if and only if there exists two integers $p, q$ such that $p q=k$ and $p+q \leq n+1$. Moreover the number of principal avalanches of size $k$ is equal to twice the number of couples 
$(p, q)$ such that $p q=k$ and $p+q \leq n$ plus the number of couples such that $p q=k$ and $p+q=n+1$.

Proof. The recurrent configurations on the cycle $C_{n+1}$ are the configurations in which all vertices have one particle except possibly one which has no particle; there are $n+1$ such configurations. The recurrent configuration is determined by the distance from the sink to the vertex with no particle and to obtain the size of the avalanche one has to consider where the particle has been added. Let us denote by $1^{n}$ the recurrent configuration consisting of a full sequence of 1 's and by $1^{p} 01^{q}$ (with $p+q=n-1$ ) those containing $n-1$ 1's and one 0 .

It is easy to check that the avalanche starting with $1^{p-1} 21^{q-1}$ has size $p q$ and the same is true for the avalanche starting with the configuration $1^{p-1} 21^{q-1} 01^{r}$ or with configuration $1^{r} 01^{p-1} 21^{q-1}$ giving the result.

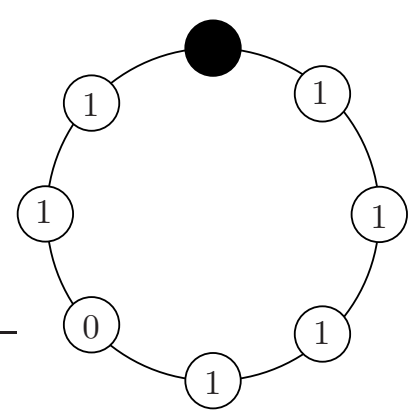

Figure 7: A recurrent configuration on $C_{8}$.

Note that the number of couples $\left(u, x_{i}\right)$ such that $u_{i}=0$ is $n$, hence the constant term of $A v_{C_{n+1}}$ is $n$.

The avalanche polynomial of $C_{8}$ is given by

$A v_{C_{8}}=7+2 x+4 x^{2}+4 x^{3}+6 x^{4}+4 x^{5}+8 x^{6}+2 x^{7}+4 x^{8}+2 x^{9}+4 x^{10}+6 x^{12}+2 x^{15}+x^{16}$

For instance the number of avalanches of size 6 is 8 since there are 4 couples $(p, q)$ with $p q=6$ and $p+q \leq 7$ namely: $(1,6),(6,1),(2,3),(3,2)$; the number of avalanches of size 16 is 1 since the couple $(4,4)$ is the only one satisfying $p q=16$ and $p+q=8$ and there is no $p, q$ satisfying $p q=16$ and $p+q \leq 7$.

\section{Avalanche polynomials of the complete graph $K_{n+1}$}

In the complete graph $K_{n+1}$ the recurrent configurations are in bijection with parking functions. Recall that a sequence of non negative numbers $\left(w_{1}, w_{2}, \ldots, w_{n}\right)$ is an $n$-parking function if there exists a permutation $a_{1}, a_{2}, \ldots, a_{n}$ of $1,2, \ldots, n$ such that for all $i, 0 \leq w_{i}<a_{i}(\operatorname{cf}[12])$. For instance the 163 -parking functions are the permutations of the following sequences:

$$
(0,1,2),(0,0,1),(0,1,1),(0,0,2),(0,0,0) .
$$

A simple use of Proposition 1 gives 
Proposition 3. The configurations $u_{1}, u_{2}, \ldots, u_{n}$ of $K_{n+1}$ is recurrent, if and only if $n-1-u_{1}, n-1-u_{2}, \ldots, n-1-u_{n}$ is a parking function. Then the number of recurrent configurations on $K_{n+1}$ is $(n+1)^{n-1}$.

Moreover in this correspondence the number of saturated vertices in $u$ is equal to the number of 0 in the associated parking function.

The number of avalanches of size 0 in $K_{n+1}$ is equal to the number of non zero elements in all the $n$-parking functions. To compute this number we need the following lemma (cf [1]):

Lemma 1. The number of parking functions containing $k$ O's is :

$$
\left(\begin{array}{l}
n-1 \\
n-k
\end{array}\right) n^{n-k}=\frac{k}{n}\left(\begin{array}{l}
n \\
k
\end{array}\right) n^{n-k}
$$

Proof. Consider the set $U_{n}$ of all sequences $u$ of $n$ integers containing $k$ 's and $n-k$ numbers $1 \leq u_{i} \leq n$, clearly there are $\left(\begin{array}{l}n \\ k\end{array}\right) n^{n-k}$ such sequences. All parking functions are in $\bar{U}_{n}$ but the converse is not true. To determine if $u \in U_{n}$ is a parking function we apply the following parking algorithm:

Consider a park place with $n$ slots numbered $1,2, \ldots, n$ lying on a circle. For each $i$ such that $u_{i}>0$ put a car on the first free slot starting from position $u_{i}$ and going around the circle.

The sequence $u$ is a parking function if and only if the slot $n$ is free at the end of the algorithm.

For instance the algorithm applied to the sequence 2,5,0,5,5,0,0 fills the slots $2,5,6,7$, and the slots $1,3,4$ are free; the sequence is not a 7 -parking function.

After the execution of the algorithm there are $k$ free slots, and by symmetry each of the slots $1,2, \ldots, n$ has an equal probability of being free; hence the probability of the slot $n$ to be free is $\frac{k}{n}$, giving the result.

From the above Lemma we obtain;

Proposition 4. The number of principal avalanches of size 0 in $K_{n+1}$ is

$$
n(n-1)(n+1)^{n-2} \text {. }
$$

Proof. It suffices to check that the number of couples $\left(u, x_{i}\right)$ such that $u$ is an $n$ parking function and $u_{i}=0$ is equal to $2 n(n+1)^{n-2}$; the result is then obtained by subtracting this value from the total number of couples $\left(u, x_{i}\right)$ which is $n(n+1)^{n-1}$. The number of the couples we are considering is by the Lemma above

$$
T_{n}=\sum_{k=1}^{n-1} k\left(\begin{array}{l}
n-1 \\
n-k
\end{array}\right) n^{n-k} .
$$

Denote $f_{n}(x)=x(n+x)^{n-1}$ we have:

$$
f_{n}(x)=\sum_{k=0}^{n-1} x^{k} n^{n-k}\left(\begin{array}{l}
n-1 \\
n-k
\end{array}\right),
$$

and

$$
T_{n}=f_{n}^{\prime}(1)=(n+1)^{n-1}+(n-1)(n+1)^{n-2},
$$

giving the result 

have:

In order to enumerate the number of principal avalanches of positive sizes we

Proposition 5. The number of principal avalanches of size $m>0$ in $K_{n+1}$ is:

$$
\left(\begin{array}{l}
n \\
m
\end{array}\right) m^{m-1}(n-m+1)^{n-m-1}=n\left(\begin{array}{c}
n-1 \\
m-1
\end{array}\right) m^{m-2}(n-m+1)^{n-m-1} .
$$

Proof. In order to prove this result we associate a subset and two different recurrent configurations to any couple $\left(u, x_{i}\right)$ consisting of a recurrent configuration $u$ and a saturated site $x_{i}$ which gives a principal avalanche of size $m>0$ when a particle is added on site $x_{i}$ :

- a subset $J$ of $m-1$ sites among the $n-1$ sites of $K_{n+1}$ different from the sink and from $x_{i}$ : these are the sites which perform a toppling during the avalanche triggered by the toppling of $x_{i}$,

- a recurrent configuration on $K_{m}$ : consider the values $u_{j}$ for $x_{j} \in J$ and subtract $m-2$ to all these values; it is easy to check using Proposition 1 that it is a recurrent configuration,

- a recurrent configuration on $K_{n-m+1}$ : the values $u_{k}$ for $x_{k} \notin J \cup\left\{x_{i}\right\}$ determine a recurrent configuration on this graph.

Conversely it is easy to build a principal avalanche of size $m>0$ from a subset $J$ of $m-1$ sites, a vertex $x_{i}$, a recurrent configuration on $K_{m}$ and another one on $K_{n-m+1}$.

Below is given the sizes of avalanches for the complete graph $K_{5}$. The recurrent configurations which differ by a permutation of the number of particles on the sites are considered as equivalent. For $K_{5}$, we represent each equivalence class by the configuration satisfying; $u_{1} \leq u_{2} \leq \ldots \leq u_{n}$, we also give the class sizes and the number of principal avalanches of each size.

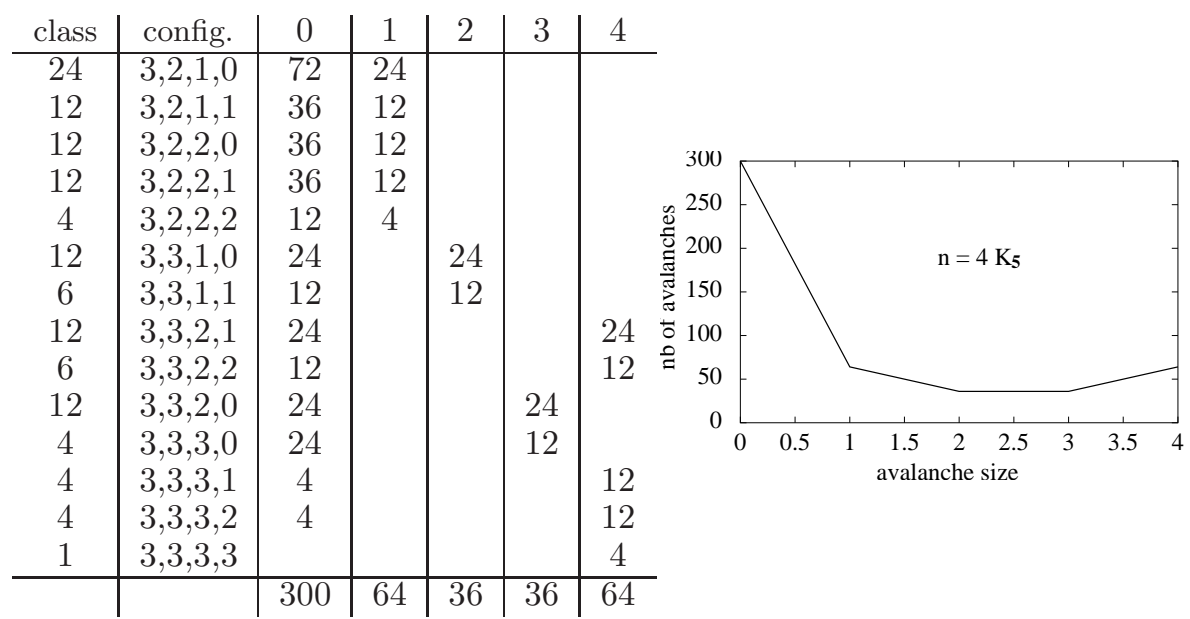


The distribution of avalanches sizes of the graph $K_{21}$ are given in Figure 8 .

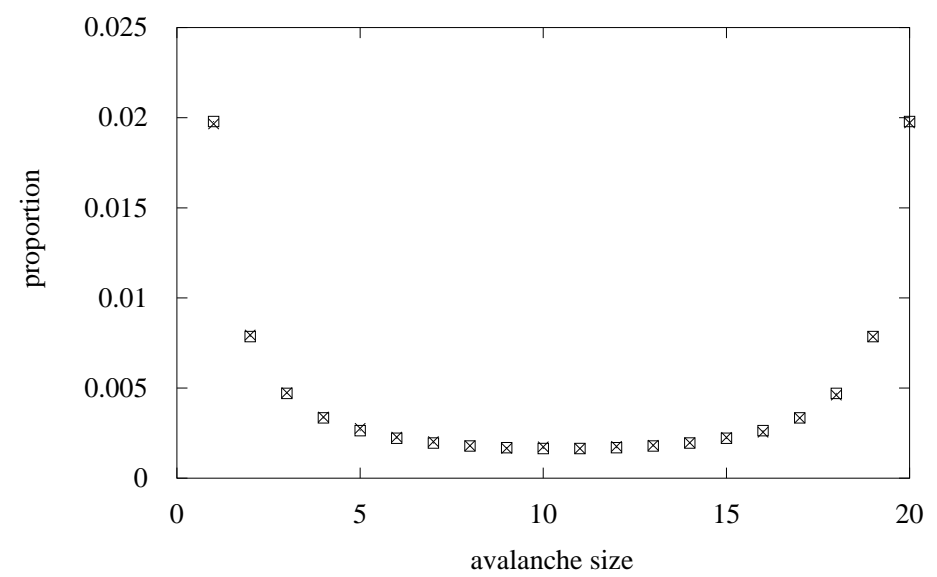

Figure 8: Distribution of principal avalanches on $K_{21}$ : theoretical prediction (square) and experiments (cross).

\section{Avalanche polynomials of lollypop graphs}

In this section we consider avalanches on the lollypop graph with $n+m+1$ vertices. It consists of a path of vertices $x_{0}, x_{1}, x_{2}, \ldots, x_{m}$ leading to the complete graph $K_{n+1}$ whose vertices are $x_{m}, x_{m+1}, \ldots, x_{m+n}$. It is more convenient to consider $x_{0}$ as the sink. In other words, we apply $\phi^{m}$ to $K_{n+1}$.

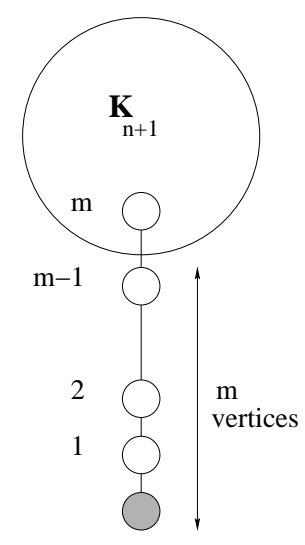

Figure 9: Lollypop graph $L_{m, n}$.

A recurrent configuration on this graph consists of a recurrent configuration

$$
u_{m+1}, u_{m+2}, \ldots, u_{m+n}
$$


on the complete graph, where the other vertices are saturated. This gives:

$$
u_{m}=n, u_{m-1}=1, \ldots, u_{1}=1 .
$$

The sizes of the principal avalanches are given by:

Proposition 6. In the lollypop graph the sizes of the principal avalanches of the recurrent configuration $u$ are

- 0 , if the particle is added on a non saturated vertex for $u$,

- $i(m+n)-\frac{i(i-1)}{2}$, if the particle is added to the $i$-th vertex of the path $(i \leq m)$ starting from the sink,

- $\left[\frac{m(2 n+m+1)}{2}+1\right] a d_{K_{n+1}}\left(u, x_{i}\right)$, if the particle is added in a saturated vertex $x_{i}$ for $u$.

As an example the avalanche polynomial of the lollypop graph $L_{4,3}$ is :

$$
24+16 x^{7}+16 x^{13}+16 x^{18}+16 x^{22}+9 x^{23}+6 x^{46}+9 x^{69} .
$$

A computation of the sizes of principal avalanches for the lollypop graph $L_{10,20}$ gives the distribution shown in the Figure below:

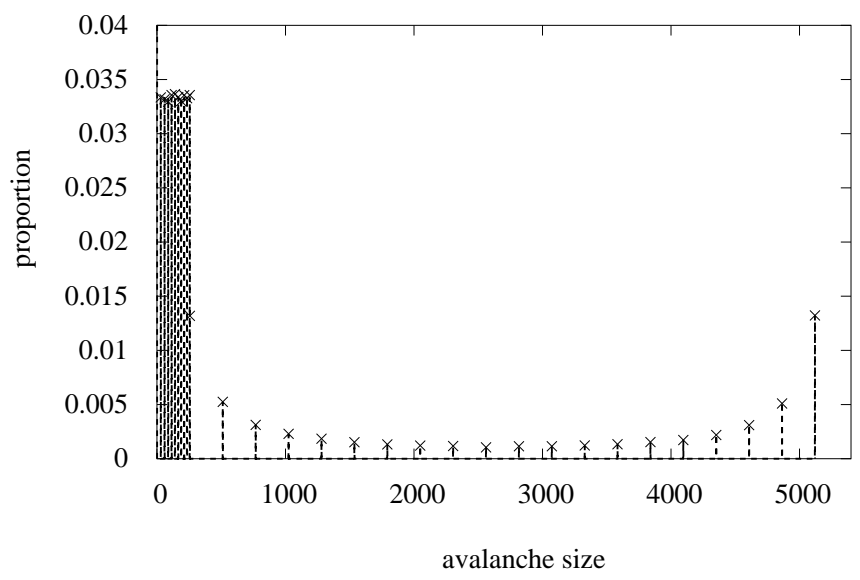

Figure 10: Avalanche distribution for $L_{10,20}$ : expected result (dashed line), and experiments over $10^{6}$ computations (cross).

Let us define the operator $\operatorname{Tr}^{k}$ for every polynomial $P=\sum_{i=0}^{n}$ as follows:

$$
\operatorname{Tr}^{k}(P)(x)=\sum_{i=0}^{\min (k, n)} a_{i} x^{i}
$$


Corollary 1. The avalanche polynomial of the lollypop graph $L_{m, n}$ is:

$$
A v_{L_{m, n}}(x)=A v_{K_{n+1}}\left(x^{a+1}\right)+(n+1)^{n-1} \operatorname{Tr}^{a}\left(A v_{L_{m+n}}\right)(x),
$$

where $a=\frac{m(2 n+m+1)}{2}$ and $L_{k}$ is the line with $k+1$ vertices.

Proof. The first term of the sum is obvious by the precedent proposition. The second term corresponds to principal avalanches for couples $\left(u, x_{i}\right)$, where $i \leq m$, i.e. where $x_{i}$ is a vertex of the path $L_{m}$. The size of this principal avalanche is the same if the vertices $x_{j}$ for $j>i$ are all in line. Indeed, what matters when a particle is added on $x_{i}$ is how many vertices are in the subgraph when deleting the path $x_{0}, x_{1}, \ldots, x_{i}$. For $i \leq m$, the avalanche size is at most $a$, thus $\operatorname{Tr}^{a}\left(A v_{L_{m+n}}\right)(x)$ gives the good exponents. Since there are $(n+1)^{n-1}$ recurrent configurations, we get the result.

In fact, this result could be generalized. Let $G=(X, E)$ be a rooted graph. We call dissipation of a principal avalanche the number $d$ of particles that the sink (root) receives during the avalanche. We associate a new polynomial enumerating the principal avalanches by size and dissipation to any graph $G$; this polynomial is given by:

$$
\widetilde{A v_{G}}(x, y)=\sum \alpha_{k, d} x^{k} y^{d}
$$

where $\alpha_{k, d}$ is the number of principal avalanches of size $k$ and dissipation $d$.

If $G$ is a tree, $\widetilde{A v_{G}}(x, y)$ has a very simple expression: $\widetilde{A v_{G}}(x, y)=A v_{G}(x) y$. Indeed, every principal avalanche has dissipation 1.

If $G$ is such that every vertex is connected to the sink, like $K_{n+1}$ for example, $\widetilde{A v_{G}}(x, y)$ has also a very simple expression: $\widetilde{A v_{G}}(x, y)=A v_{G}(x y)$. Every principal avalanche of size $m$ admits $m$ as dissipation, since every toppling gives a particle to the sink.

Then, if we apply $\phi^{m}$ to $G$ like we did with $K_{n+1}$ to obtain a lollypop graph, we get a graph $G_{m}$, whose avalanche polynomial is:

$$
A v_{G_{m}}(x)=\widetilde{A v_{G}}\left(x, x^{a}\right)+\frac{A v_{G}(1)}{n} \operatorname{Tr}^{a}\left(A v_{L_{m+n}}\right)(x),
$$

where $a=\frac{m(2 n+m+1)}{2}$ and $|G|=n+1$. In fact we have:

$$
\widetilde{A v_{G_{m}}}(x, y)=\widetilde{A v_{G}}\left(x, x^{a} y\right)+\frac{A v_{G}(1)}{n} \operatorname{Tr}^{a}\left(A v_{L_{m+n}}\right)(x) y \text {. }
$$

For the lollypop graph, it is particulary simple, since $\widetilde{A v_{K_{n+1}}}(x, y)=A v_{K_{n+1}}(x y)$.

\section{Concluding remarks}

In this paper, we have considered avalanche polynomials which encode the distribution of the sizes of avalanches. We have seen that for the complete graph, we have a huge peak for size 0 . Now the tree $T_{n}$ defined on Figure 11 gives a peak for size $n$.

The construction consisting of merging the sinks of two rooted graphs translates in adding the avalanche polynomials. Applying this construction to the two 


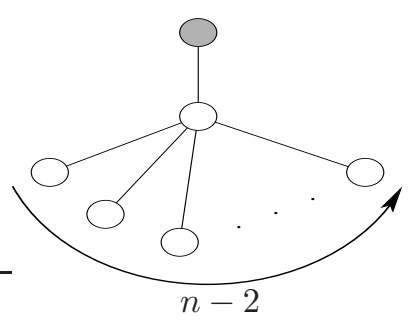

Figure 11: Tree $T_{n}$.

kind of graphs considered above, we are able to build graphs having any shape of distribution in a certain sense.

This informal remark can be formalized in a more precise result which will be developped in a future work.

\section{Acknowledgement}

We thank Michel Marcus for his example.

\section{References}

[1] P. Bak. How nature works - the science of soc. Oxford university press, 1997.

[2] P. Bak, C. Tang, and K. Wiesenfeld. Self-organized criticality: an explanation of $1 / f$ noise. Phys. Rev. Lett., 59, 1987.

[3] N. L. Biggs. Chip-firing on distance-regular graphs. Tech. Report LSE-CDAM96-11, CDAM Research Report Series, June 1996.

[4] N. L. Biggs. Chip-firing and the critical group of a graph. Journal of Algebraic Combinatorics, 9(1):25-45, 1999.

[5] K. Chen, P. Bak, and S. P. Obukhov. Self-organized criticality in a crack propagation model of earthquakes. Physical Review Letters A, 43:625-30, 1991.

[6] R Cori and D Rossin. On the sandpile group of dual graphs. European J. Combin., 21(4):447-459, 2000.

[7] M. Creutz. Abelian sandpile. Computers in Physics, 5:198-203, 1991.

[8] A. Dartois and D. Rossin. Analysis of the distribution of the length of avalanches on the sandpile group of the $(n, k)$-wheel. FPSAC, 2003.

[9] D. Dhar. Self-organized critical state of sandpile automaton models. Physical Review Letters, 64:1613-1616, 1990.

[10] D. Dhar, P. Ruelle, S. Sen, and D. Verma. Algebraic aspects of abelian sandpile models. Journal of Physics A, 28:805-831, 1995. 
[11] Dominique Foata and John Riordan. Mappings of acyclic and parking functions. Aequationes Math., 10:10-22, 1974.

[12] A. G. Konheim and B. Weiss. An occupancy discipline and applications. SIAM J. Appl. Math., 1966.

[13] D. V. Ktitarev, S. Lübeck, P. Grassberger, and V. B. Priezzhev. Scaling of waves in the bak-tang-wiesenfeld sandpile model. Physical Review E, 61:8192, 2000 .

[14] A. Sornette and D. Sornette. Self-organized criticality of earthquakes. Europhys. Lett., 9:197-202, 1989.

\section{Cori, Robert}

LABRI, Université Bordeaux 1

cori@labri.u-bordeaux.fr

Dartois, Arnaud

LIX, École Polytechnique

dartois@lix.polytechnique.fr

Rossin, Dominique

CNRS and LIAFA, Université Paris 7

rossin@liafa.jussieu.fr 